\title{
Light impurity transport at an internal transport barrier in Alcator C-Mod
}

\author{
William L. Rowan ${ }^{1}$, Igor O. Bespamyatnov ${ }^{1}$ and C.L. Fiore ${ }^{2}$ \\ ${ }^{1}$ Fusion Research Center, The University of Texas at Austin, Austin, TX 78712, USA \\ ${ }^{2}$ MIT Plasma Science and Fusion Center, Cambridge, MA 02139, USA \\ E-mail:w.1.rowan@mail.utexas.edu
}

Received 15 April 2008, in final form 21 August 2008

Published 10 September 2008

Online at stacks.iop.org/NF/48/105005

\begin{abstract}
Density profiles for a light impurity, boron, are reported for internal transport barrier (ITB) discharges in Alcator C-Mod. During the ITB, the light impurity gradient steepens because the impurity pinch increases relative to diffusion. The ITB-induced impurity profile steepening is at approximately the same major radius as that for the main-ion profile. Neoclassical transport does not describe the light impurity profiles but transport is closer to neoclassical in the ITB region. In previous work on C-Mod, profiles of seeded heavy impurities (introduced by puffing) peaked during the ITB, but a marked difference between transport of heavy and light impurities has been reported for other tokamaks. With the addition of light impurity profiles described here, the ITB on C-Mod is shown to share additional profile traits with the ITB on other tokamaks. This confirms that the macroscopic features of the C-Mod ITB are similar to those on other devices although it leaves open the details of the onset of the ITB.
\end{abstract}

PACS numbers: $52.25 . \mathrm{Fi}, 52.25 . \mathrm{Vy}, 52.55 . \mathrm{Fa}$

\section{Introduction}

Tokamak discharges with internal transport barriers (ITBs) have peaked main-ion profiles that may lead to higher core reactivity. If the impurity profile is also peaked or if impurities simply accumulate, then there will be deleterious effects on the plasma: radiation losses will increase and plasma dilution will reduce reactivity. New measurements of boron profiles with spatial and temporal resolution are reported for Alcator C-Mod. With the addition of these light impurity profiles, the ITB phenomenological description in C-Mod is more complete and more easily compared with the ITBs generated in other tokamaks. In view of the differences between light and heavy impurity transport in ITBs [1], it is important to characterize the behaviour of light impurities.

The Alcator C-Mod ITB is described in detail elsewhere $[2,3]$. Only a brief summary is included here as a background for this research. Typically, an ITB can be formed only from enhanced $\mathrm{D}_{\alpha}$ (EDA) H-Mode plasmas. Off-axis deposition of ion cyclotron resonance heating (ICRH) triggers the ITB described here, but an ITB can also be formed without any auxiliary heating by applying a particular prescription for toroidal field ramping. The ITB is known to be characterized by peaked pressure profile which is mainly a result of peaking in the main-ion density profile. Thermal diffusivity $\chi_{\mathrm{eff}}$ is reduced during the ITB as evidenced by profile simulation [2] and by heat pulse propagation [4]. $T_{\mathrm{e}}$ does not peak as shown in those references and here as well. $\left(T_{\mathrm{i}}, T_{\mathrm{e}}\right.$ and $n_{\mathrm{e}}$ peaking as observed in C-Mod and in other devices is discussed in a recent review [5].) Heavy impurities (argon) accumulate in the ITB. The density peaking can lead to excitation of a trapped electron mode (TEM) which quenches the peaking in the ITB [6]. The TEM can be controlled by heating the core with additional ICRH. Further peaking of the pressure profile is then quenched, and a quasi-stationary profile is maintained. Finally, note that the ITB is a macroscopic feature which is not necessarily due to the same mechanisms on different devices. Thus, this paper adds missing data, light impurity profiles, to the other Alcator C-Mod data and may lead to improved understanding of ITBs in C-Mod.

We have extended the work of Rice [3] for heavy ions to lighter, boron ions. In the earlier work on C-Mod, chordaveraged measurements of puffed argon ions were reproduced in numerical simulations of impurity transport to characterize the transport. The measurements described here are made using charge-exchange recombination spectroscopy (CXRS) techniques with emission excited by a diagnostic neutral beam (DNB) since the ions of principal interest are fully stripped and good spatial resolution is required. An advantage in the study of light ions is that the fully stripped ion may contain the entire density of the impurity in high temperature tokamaks such as C-Mod. Analysis and interpretation is then less complicated. 
The usual numerical analysis is simpler. Useful transport information can also be extracted from analytical analysis of the profiles. The latter is not possible for heavy impurities. These advantages of light impurity ions have been effectively exploited on other devices $[7,8]$.

In previous work on the Joint European Torus (JET) [1], and for ITB discharges on JT-60 [8], it was demonstrated that light impurity transport differs from heavy impurity transport. Light impurity transport has not yet been reported for C-Mod. Here, light impurity transport is added to the data previously taken on C-Mod and provides another point of comparison for ITBs in different devices. Completing these sets of profiles for different devices is important in that the ITB may differ among devices.

The paper is organized as follows. Section 2 describes the plasma conditions, the magnetic configuration that is used to generate the ITB in Alcator C-Mod and the methods for measurement of the boron density profiles. Section 3 introduces the measured profiles of boron. In particular, the gradient steepening in the impurity profiles which are caused by the ITB are compared with the ITB-induced effects in the $T_{\mathrm{i}}, n_{\mathrm{e}}$ and $T_{\mathrm{e}}$ profiles. The impurity transport analysis is presented in section 4 . The analysis of the fully stripped profile of boron in section 4 demonstrates that the impurity pinch is enhanced during the ITB. The changes in the convection are more than a simple change in magnitude: evidence is presented for a change in direction of the impurity convection. Just prior to the formation of the ITB, the convection is outwards. As the ITB evolves, the convection becomes an inward pinch which increases in time before reaching a saturation. This is confirmed by numerical impurity transport simulations. Comparison with the benchmark neoclassical transport indicates that anomalous transport of light impurities is reduced during the ITB and at the location of the ITB. Comparison with heavy impurity C-Mod results and with light and heavy impurity measurements on other devices is presented in section 5 .

\section{Experimental conditions}

The ITB for these experiments was formed in a plasma of constant toroidal field (4.4 T) and constant plasma current (0.8 MA). The magnetic configuration was a lower single null with $\vec{B} \times \nabla B$ in the favourable direction. The EFIT [9] reconstruction is in figure 1. Safety factor $q_{95}$ for these discharges was approximately 4.1. Typical time series for these discharges are shown in figure 2. Auxiliary ICRH heating (figure 2, panel a) with an input power of $4 \mathrm{MW}$ triggered the ITB at approximately $0.73 \mathrm{~s}$. The resonance locations were on the high field side as indicated in figure 1 . The deposition profile is shown in figure 3. Typical core electron density was $4.0 \times 10^{20} \mathrm{~m}^{-3}$. Following ITB trigger, the $n_{\mathrm{e}}$ profile evolved to a peaked profile as shown in figure 2, panel $\mathrm{d}$. The core electron temperature was $1.5 \mathrm{keV}$. The boron density peaked also following onset of the ITB (figure 2, panel f). Vertical lines in figure 2 show the times for which profiles will be reported. Profile peaking begins soon after the time indicated by the blue vertical line, and this time is labelled as $\Delta t=0$ in the profile graphs.

The density profiles of intrinsic boron are measured with active charge exchange. A new long-pulse (1.5 s) DNB is used

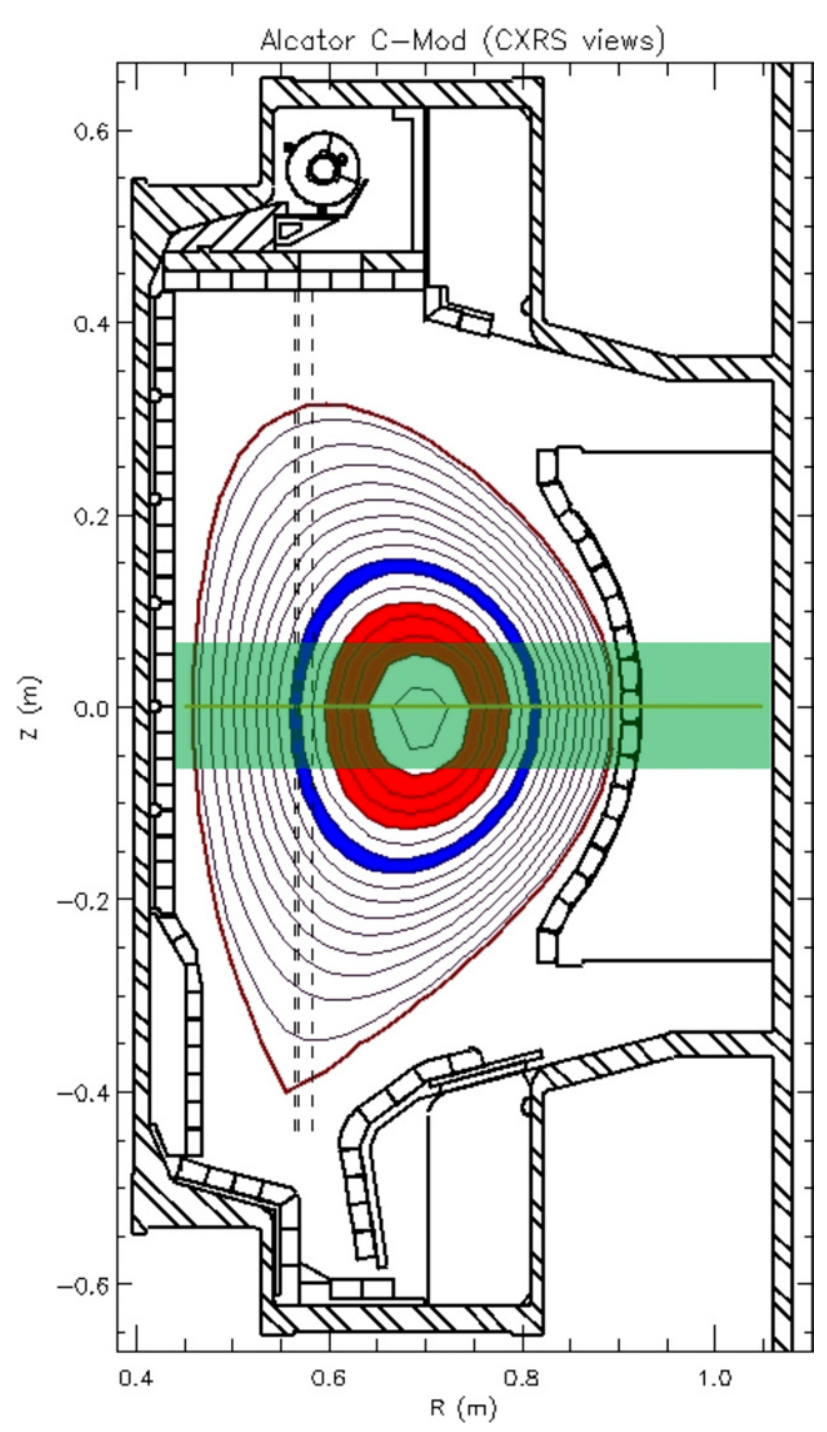

Figure 1. Typical magnetic configuration for the ITB discharges. $\vec{B} \times \nabla B$ is in the favourable direction. ICRH is resonant on the high field side of the plasma (vertical dashed lines for $R<0.6 \mathrm{~m}$ ). The DNB is displayed as a green rectangle. Improved boron confinement is observed on flux surfaces (shaded in red) which are interior to the flux surfaces on which ICRH is resonant (shaded in blue).

to measure the boron density for the full plasma pulse [10,11]. The beam is injected from the low field side of the plasma and is displayed as a green rectangle in figure 1. Its radius is approximately $6.2 \mathrm{~cm}$ (half width at half maximum) [12], and it is typically operated at $50 \mathrm{kV}$ with an accelerated current of $6 \mathrm{~A}$. The boron spectral line used is $4966.67, B^{+4}, n=6-7$. Emission is collected with a recently installed poloidal, wideview optical array [13]. The array comprises 20 viewing chords which are lined up along the beam axis and cover the outer half of the C-Mod cross-section $(0.67 \mathrm{~m}<R<0.91 \mathrm{~m})$. The channels have spatial resolution of $\pm 0.8 \mathrm{~cm}$ and are separated by $1.2 \mathrm{~cm}$ from each other. The spatial resolution includes both the curvature of the flux surfaces and the imaging properties of the viewing optics. All optical channels are absolute-intensity calibrated. The ultimate temporal resolution of the diagnostic is $12.5 \mathrm{~ms}$ which is defined by the beam modulation capability and the read-out time for the spectrally resolved images. With 


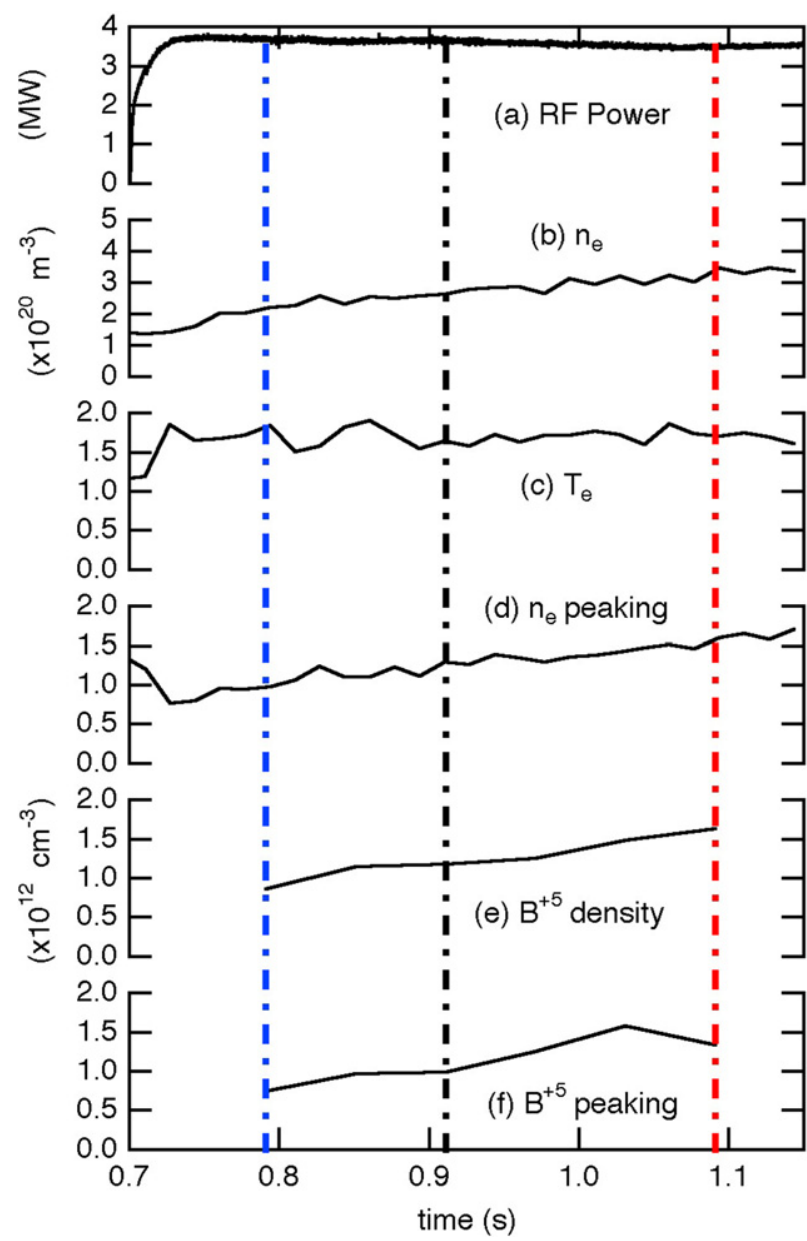

Figure 2. Time series for the ITB discharge. (a) RF power, (b) core $n_{\mathrm{e}}$, (c) core $T_{\mathrm{e}}$, (d) $n_{\mathrm{e}}$ peaking, (e) core $\mathrm{B}^{+5}$ density, (f) $\mathrm{B}^{+5}$ peaking. The principal analysis times are identified. For use in later figures: $\Delta t=0$, blue vertical line, $\Delta t=0.12$, black, $\Delta t=0.3$, red

this beam, the ratio of active to passive emission is typically $0.5-1$. The active charge-exchange signal is separated from the passive signal in the observed emission spectra by means of time-slice subtraction and the uncertainty in all data processing is included in the error bars shown in the graphs. The atomic data and the experiments used to determine contribution from excited beam neutrals are described elsewhere [14]. Beam stopping cross sections required for the analysis are from the Atomic Data and Analysis Structure (ADAS) [15] which is also reported elsewhere [16]. A second source [17] for the data has been used with no significant change in results.

\section{Measured boron profiles and comparison with other plasma profiles}

The $\mathrm{B}^{+5}$ density profile steepens during the ITB. As will be shown in section 4 , the fully stripped $\mathrm{B}^{+5}$ ion is the only boron ion for $\rho<0.7$ where $\rho$ is normalized poloidal flux, and the boron profiles in figure 3 are also total boron profiles. That fact will be used to simplify the transport analysis in section 4. The profiles in figure 3 were acquired as the boron density evolved following the formation of the ITB. The uncertainties represent statistical errors in fitting the data

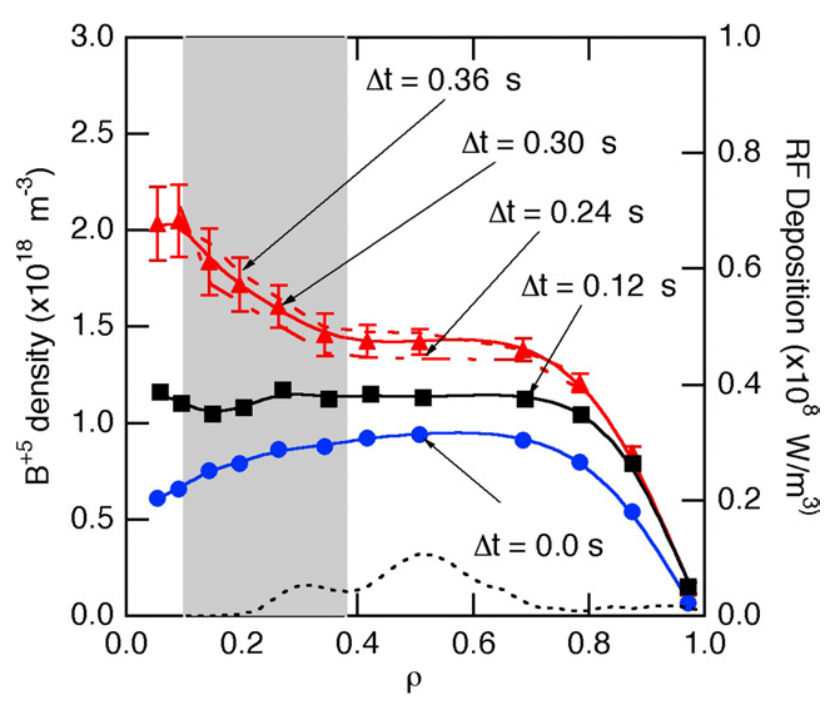

Figure 3. Evolution of $\mathrm{B}^{+5}$ profile versus normalized poloidal flux $\rho$ during an ITB. $\Delta t$ is the time elapsed following ITB formation. The ITB-induced impurity gradient is interior to the ICRH deposition (dotted black line, right axis) and interior to the ITB region as defined using the electron density profile. The uncertainties displayed for $\Delta t=0.3$ are typical of all the $\mathrm{B}^{+5}$ density data.

to a line model as well as uncertainties in the calculation of the beam density attenuation due to electron temperature and density uncertainties. The uncertainty displayed for the fully evolved ITB profile at $\Delta t=0.3 \mathrm{~s}$ are typical of all the $\mathrm{B}^{+5}$ density data shown in figure 3 . Immediately after the formation of the ITB, the boron density profile is unchanged from the EDA H-mode profile and is characterized by a slightly hollow or flat profile. As the profile evolves during the ITB, it steepens in the range $0.1<\rho<0.4$. This region is shaded in figure 3, and is the main region of interest here. The limits will be discussed later. Profile evolution ends in a quasi-stationary profile approximately $0.24 \mathrm{~s}$ after the ITB forms and is then time independent for approximately $0.12 \mathrm{~s}$. The gradient of the fully evolved profile will be shown to indicate improved confinement in section 4. As shown in figure 1, ICRH is resonant on the high field side of the plasma. Comparison with that and with the deposition profile in figure 3 shows that impurity gradient steepening is interior to the flux surfaces on which the ICRH is deposited.

The pressure profile peaks as the ITB evolves (figure 4). Density peaking is the main contributor as indicated by the electron density profile, also in figure 4. Electron temperature is less of a contributor as indicated in figure 5. The foot of the ITB is defined using the electron density profile and indicates the largest radial extent of the main-ion particle effects of the ITB. The peaking of the $n_{\mathrm{e}}$ profile occurs for $\rho<0.4$. For $\rho>0.4, n_{\mathrm{e}}(\rho)$ is unchanged by the ITB. The region of principal interest is shaded in the figures. The region is bounded at large $\rho$ by observable $n_{\mathrm{e}}$ profile effects. The region is bounded at small $\rho$ by the practical limits on analysis of boron data. The light impurity profile steepens inside the ITB region. The $T_{\mathrm{e}}$ profile is essentially unchanged during the transition to the ITB. Note also that the small change in $T_{\mathrm{e}}$ is a feature of C-Mod ITBs and does not imply a lack of improvement in the thermal channel as discussed in section 1 . 


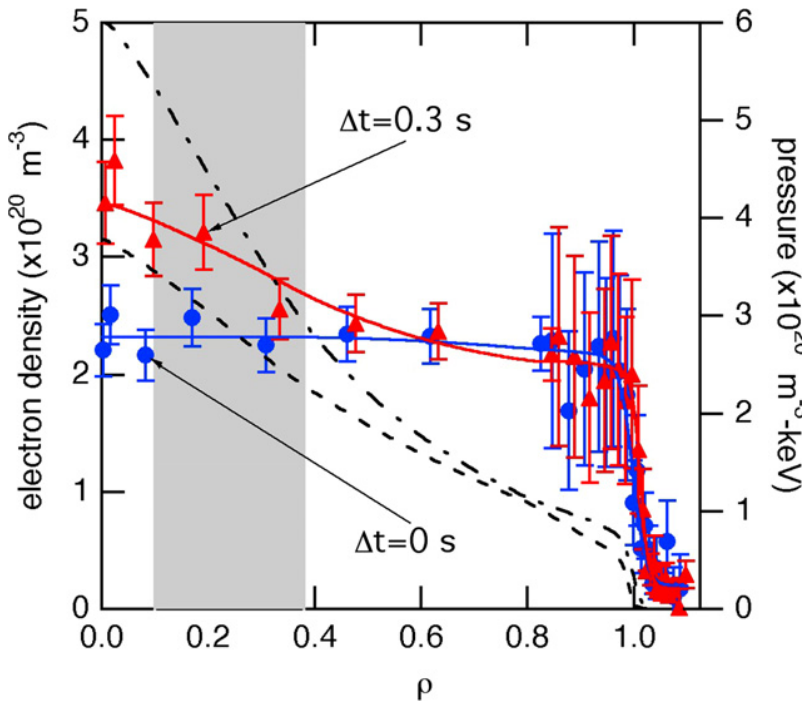

Figure 4. Electron density profile evolution during an ITB. $\Delta t$ is the time after the ITB forms. The pressure profile is also shown as dashed and dot-dash lines. The ITB foot is defined using the experimental data points and is at the right edge of the shaded region.

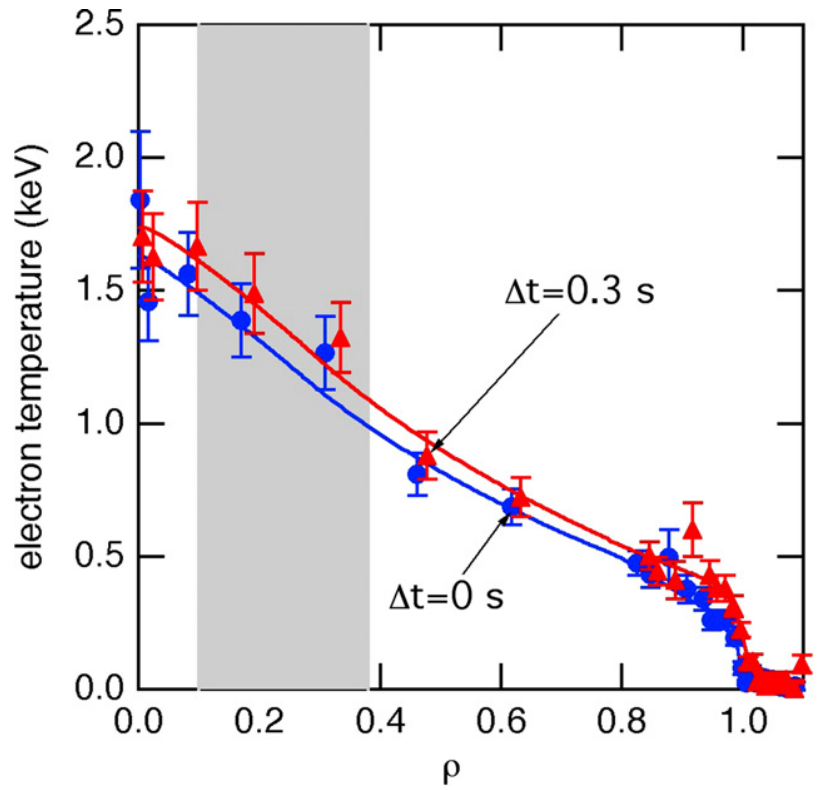

Figure 5. Electron temperature profile evolution during the ITB. $\Delta t$ is the time after the ITB forms. The ITB foot is at the right edge of the shaded region.

The boron peaking is compared with electron density peaking in figure 6. Different discharges are distinguished by colour, and the increase in peaking which occurs with time can be followed from the lower left to the upper right for each discharge. As illustrated in figure 2 and in figure 4, the electron density profile peaking increases as the ITB evolves. The comparison in figure 6 demonstrates that boron peaking starts weaker but increases more rapidly than does the electrondensity profile. The boron profile can achieve values that are more strongly peaked than the electron density profile. In these ITB discharges, accumulation is not consistently observed but may occur.

As for $T_{\mathrm{e}}, T_{\mathrm{i}}$ is little changed during the ITB although it may evolve to slightly higher values (figure 7 ). The increase

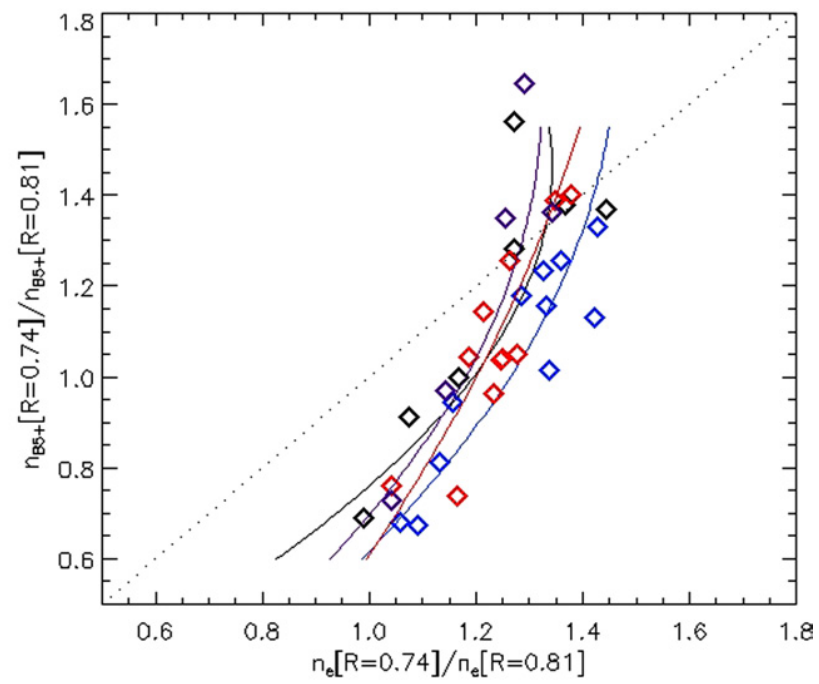

Figure 6. Peaking of the boron density profile relative to the electron density profile. Peaking is displayed for a group of closely related discharges each of which is distinguished by colour. For a particular discharge, time increases from lower left to upper right.

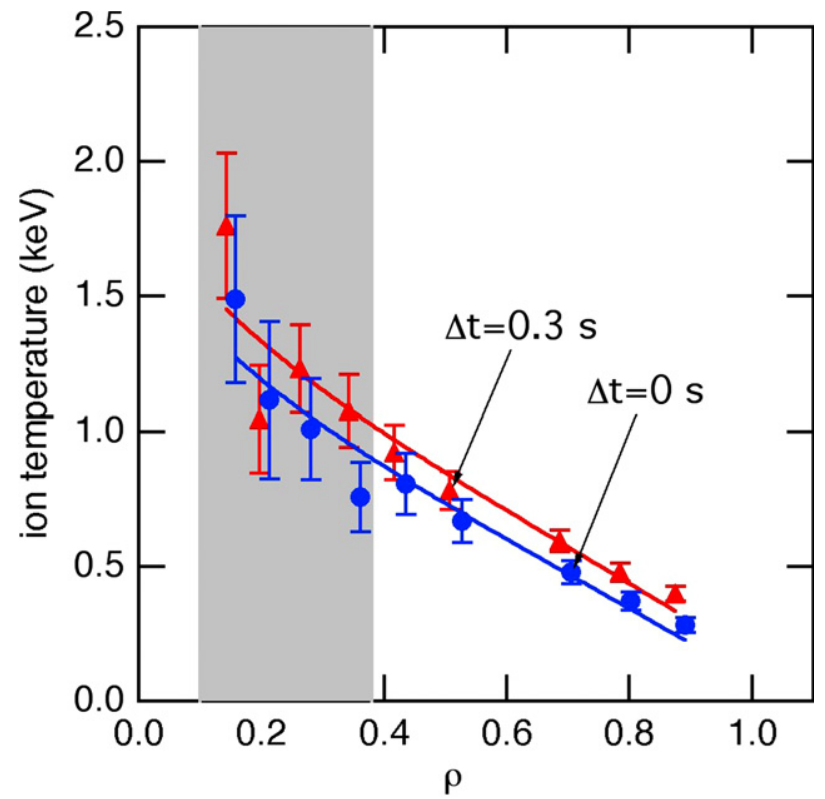

Figure 7. Ion temperature profile evolution during the ITB. $\Delta t$ is the time after the ITB forms. The ITB foot is at the right edge of the shaded region.

may be as large as approximately $20 \%$ and is consistent across the set of discharges used in this analysis and is also consistent with the increase in $T_{\mathrm{e}}$. The $T_{\mathrm{i}}$ profile may be broadening as predicted by Zhurovich [18]. Unfortunately, typical data uncertainty in this experiment precludes this conclusion. It is useful to note that broadening of the $T_{\mathrm{i}}$ profile leads to stabilization of ion temperature gradient (ITG) modes which then leads to the ITB.

\section{Impurity transport}

Impurity transport information is extracted from the data using an analytical analysis of the profiles as well as numerical 
simulation of the impurity transport. As is well known, impurity transport is governed by

$$
\frac{\partial n_{j}^{z}}{\partial t}+\nabla \cdot \Gamma_{j}^{z}=S_{j}^{z}
$$

where $n_{j}^{z}$ is the $j$ th charge state for impurity $z, \Gamma_{j}^{z}$ is the impurity flux and $S_{j}^{z}$ is the impurity source.

In figure 3 , the boron profile has evolved to the steepest gradient by $\Delta t=0.24 \mathrm{~s}$ and then is time independent for approximately $0.12 \mathrm{~s}$. This is the temporal range of interest here since it represents the fully evolved impurity profile for this ITB discharge. In equation (1), $\partial n_{j}^{z} / \partial t=0$.

A useful model for the flux in equation (1) is in equation (2)

$$
\Gamma_{j}^{z}=-D \frac{\partial n_{j}^{z}}{\partial r}+v n_{j}^{z},
$$

where $D$ is the diffusion coefficient and $v$ is the convective parameter.

This model is widely used. Though a simple form, it can be used to describe both quasilinear and neoclassical impurity transport.

For $\rho<0.7$, the $\mathrm{B}^{+5}$ density is the same as the total density of boron since the plasma temperature is sufficient for it to be the only boron charge state that is present. It is also a trace impurity. For that range of $\rho$, the source term in equation (1) is negligible because of the absence of other charge states of boron. The time derivative can be neglected as discussed earlier. Thus, the ratio of convection parameter to the diffusion is simply the logarithmic derivative of the $\mathrm{B}^{+5}$ density profile:

$$
\frac{1}{n_{j}^{z}} \frac{\partial n_{j}^{z}}{\partial r}=\frac{v}{D} \quad \text { or } \quad L_{Z}^{-1}=\frac{v}{D} .
$$

In this experiment, the time-independent profile of the impurity density allows inference of the ratio of convection to diffusion. A time dependent experiment, such as by variation of the impurity source, is needed to separate $D$ and $v$. The time dependent experiment is not currently possible on C-Mod.

The quantity $v / D$ is very useful for comparison of theory and experiment. On the experimental side, its computation is just a straightforward calculation of the profile scale length. Of course, it is the profile scale length of the total impurity density so it is most easily acquired from the profile of a fully stripped light impurity in a plasma of moderately high temperature and density. Predictions for $v / D$ due to collisional transport are naturally available from neoclassical theory. The results of several gyrokinetic turbulence simulations suggest the flux of equation (2) is a useful way of expressing the results [19], and the peaking factor appears repeatedly in that literature. In a more general sense, the peaking factor has been related to the radial electric field via the Einstein relation [20].

The impurity peaking factor $v / D$ or inverse scale length $L_{Z}^{-1}$ is plotted in figure 8 . The uncertainties in the two profiles derived from the data of figure 4 are based on an analysis similar to that of Testa [21]. The conditions for interpretation of the inverse scale length as the ratio of convection to diffusion hold at least over the spatial range of the ITB $(\rho<0.4)$. As the ITB evolves from $\Delta t=0$ to $\Delta t=0.3 \mathrm{~s}$, a strong inward

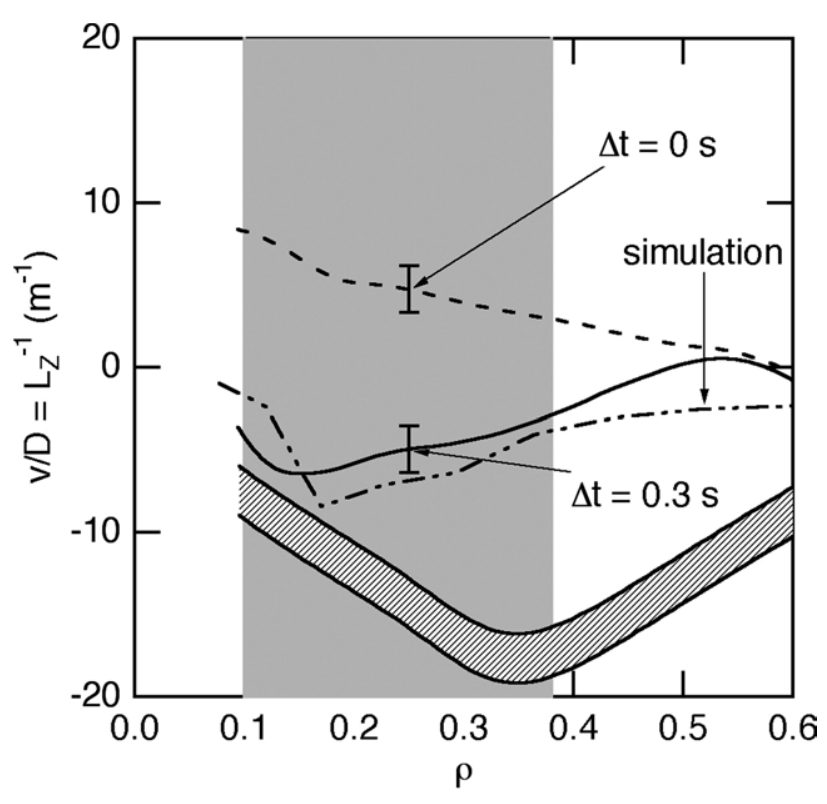

Figure 8. The inverse scale length for the impurity (solid curve and dashed curve) decreases as the ITB evolves. These are an empirical $v / D$ (interpretation motivated by equation (3)) which becomes more negative (pinch-like) in the fully developed ITB. A numerical simulation at $\Delta t=0.3 \mathrm{~s}$ is shown as a dash-dot curve. A neoclassical prediction $v_{\text {neo }} / D_{\text {neo }}$ (equation (4)) is shown as a hatched band.

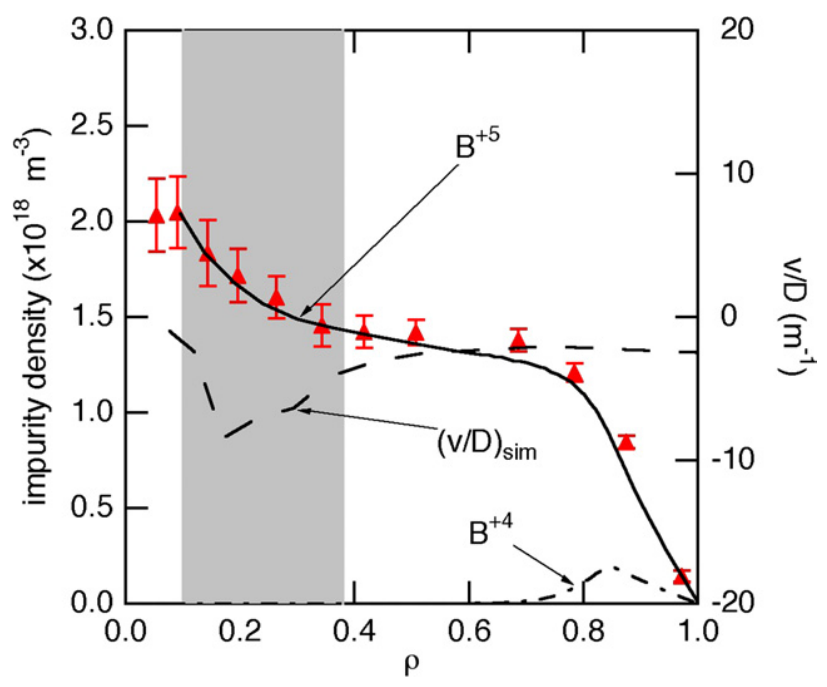

Figure 9. Simulation of $\mathrm{B}^{+5}$ density (solid line) and $\mathrm{B}^{+4}$ density (dash-dot line) compared with the measured $\mathrm{B}^{+5}$ density $(\boldsymbol{\Delta})$ at $\Delta t=0.3 \mathrm{~s} . v / D$ (dashed line) required in the simulation is plotted on the right axis.

impurity pinch appears. The pinch extends over the range $0.1<\rho<0.4$.

Numerical simulation [22] of the impurity transport for these discharges confirms the results of the profile analysis. The simulation is a $1 \mathrm{D}$ transport code in which main-ion density and temperature profiles are fixed and one impurity (boron) is allowed to reach equilibrium for a particular set of $D$ and $v$. All boron charge states are included in this simulation. The result of the simulation is shown in figure 9 for the $\Delta t=0.3$ case. $\mathrm{B}^{+4}$ is limited to the range $\rho>0.7$. For $\rho<0.7$, the only boron ion is fully stripped $\mathrm{B}^{+5}$ so 
that it represents the total boron density. Also for this range, atomic rates which are included in the transport simulation are negligible. Then, the assumptions of the profile analysis are confirmed. The simulated boron density reproduces the measured $\mathrm{B}^{+5}$ profile for the $v / D$ profile shown in the figure. The $v / D$ profile in figure 9 is the simplest that reproduces the impurity profile. Little physical significance attaches to the high spatial frequencies in the profile. The natural smoothing in the simulation would produce the same $\mathrm{B}^{+5}$ profile for smoother and more physical versions of $v / D$, but the only purpose here is to assure that a standard simulation produces the $v / D$ independently of the profile analysis described above. The simulated $v / D$ profile is reproduced in figure 8 . It is quite similar to the empirical $v / D$ and confirms that the convection becomes strongly inwards during the ITB.

The base-level transport in a tokamak is neoclassical transport. For this study as for previous work, nearness to neoclassical transport is then taken as a measure of the reduction of anomalous transport. Both diffusion and convection are a combination of anomalous and neoclassical components. Turbulence causes departures of convection from the neoclassical values (see [19] and references therein) as well as for diffusion. Since $v / D$ is the strongest result of a time-independent impurity transport experiment, nearness to neoclassical theory is quantified using this difference $\left(v_{\text {neo }} / D_{\text {neo }}\right)$

An analytical representation, equation (4), for the neoclassical ratio was developed based on $[23,24]$ :

$$
\left(\frac{v_{\text {neo }}}{D_{\text {neo }}}\right)=\frac{Z_{\mathrm{I}}}{Z_{\mathrm{D}}}\left(\frac{1}{n_{\mathrm{D}}} \frac{\mathrm{d} n_{\mathrm{D}}}{\mathrm{d} r}+K \frac{1}{T_{\mathrm{D}}} \frac{\mathrm{d} T_{\mathrm{D}}}{\mathrm{d} r}\right) .
$$

This assumes impurity-main-ion collisions dominate other collisions and that the main-ion and impurity temperatures are the same. Both are satisfied here. Electron effects do not appear because of small impurity-electron collisionality. The factor $K$ in the temperature gradient term may change sign depending on regime. For the ITB period of the discharges described here, the deuterium fuel ion is in the banana regime and boron, the impurity is in the plateau regime. For this plasma, $K=-0.18$. Whether the convection is inwards or outwards depends on a competition between density gradient term and the temperature term.

For boron, the neoclassical transport $v / D$ ratio and the measured ratio are plotted in figure 8 . The neoclassical result is plotted as a band with the width of the band following from uncertainties in the data. A pinch is predicted by neoclassical transport, and qualitatively, this is observed in experiment. Transport is closest to neoclassical in the smaller major radius portion of the ITB though the transport of the light impurity still departs from the neoclassical prediction by a significant margin. This implies that the turbulence is only partially suppressed. Partial suppression is of considerable interest as this may be the means for avoiding some to the deleterious effects of profile peaking. In fact, it has been demonstrated in C-Mod as evidenced by successful control of the ITB induced peaking by controlling core turbulence.

\section{Comparison with previous results and summary}

With this work, light impurity behaviour is added to the collection of results for C-Mod ITBs. The main features of boron transport in an Alcator C-Mod ITB are:

(a) In the region of an ITB in Alcator C-Mod, boron peaks. The hollow or flat profile observed in EDA H-mode evolves to the profile for the ITB in which the local boron density exceeds that in the plasma region outside the ITB.

(b) Boron accumulates in the ITB region only for some discharges. Accumulation of light impurities is not a consistently observed feature. For that reason, it deserves attention in future experiments.

(c) An inward pinch appears during the ITB. The pinch strengthens relative to the impurity diffusion during the evolution of the ITB.

(d) Anomalous transport is reduced during the ITB though neoclassical transport is not sufficiently dominant to predict either the impurity peaking or scale length of the gradients.

(e) Light impurity transport shares two important features with heavy impurity transport: both peak during the ITB, and the peaking is due to enhanced inward convection.

The observed peaking of light impurities is consistent with observations of ITBs on other devices $[1,25]$. When impurity profiles are compared with result of Chen [25], this Alcator C-Mod discharge appears to be similar to the strong ITB in JET. Strong and weak ITBs in JET are distinguished by the ITG, but the impurity profiles have a distinctive behaviour as well. In the strong JET ITB, core peaking results in a local core impurity density that exceeds the density in the plasma region outside the ITB. Note that the C-Mod ITB impurity profile has this same trait. The C-Mod impurity profile evolves through a state similar to the weak ITB in JET before reaching the final state. Boron accumulates in these discharges in both devices. Accumulation of light impurities was not observed in JT-60 discharges [26], and is not consistently observed on C-Mod.

The peaking of the impurity profile during the ITB is due to enhanced inward convection. This is consistent with previous results $[1,25]$ including previous work on heavy impurity behaviour on Alcator C-Mod [27]. Anomalous transport is reduced during the ITB, but neoclassical transport does not seem to be the dominant transport process even for the fully evolved ITB observed in this experiment. It is evident then that in this plasma, turbulence is only partially suppressed. A state of partial suppression of turbulence is extremely interesting as evidenced by successful control of the C-Mod ITB by inducing TEMs, and this regime is possibly worthy of further study.

Heavy impurity behaviour in C-Mod was characterized previously [3]. In that work, argon density profiles were steepened in the ITB region as the boron profiles are here. The $v / D$ ratio for argon in those discharges was much larger than for boron in the present experiment. To this must be added the caveat that the density peaking in the earlier work was larger than that produced by the ITB in these studies.

There is evidence that the impurity convection changes direction from outwards in the core of the EDA H-mode to inwards in the fully developed ITB. The reversal is not sudden change but follows from a slow evolution. Quasilinear theory for impurity convection and for the ratio, $v / D$, which is measured here can predict either an inward pinch or outward convection [19]. The pinch direction that is predicted depends on whether ions or electrons carry the turbulence. This suggests an interesting focus for future experiments though it cannot be pursued here. 


\section{Acknowledgments}

The authors gratefully acknowledge discussions with Wendell Horton, Institute for Fusion Studies, The University of Texas. They acknowledge discussions with Emilia Solano, EFDAJET, Culham Science Centre, UK, Hideo Sugama, National Institute for Fusion Science, and Richard Hazeltine, Institute for Fusion Studies, The University of Texas, on neoclassical transport topics. They also acknowledge helpful discussions with Roger Bengtson, Fusion Research Center, The University of Texas at Austin.

This work was supported by the USDOE Grant DE-FG03-96ER54373 and Coop. Agree. No DE-FC02-99ER54512.

\section{References}

[1] Dux R., Giroud C. and Zastrow K.D. 2004 Nucl. Fusion 44 260-4

[2] Fiore C.L. et al 2004 Phys. Plasmas 11 2480-7

[3] Rice J.E. et al 2002 Nucl. Fusion 42 510-9

[4] Wukitch S.J. et al 2002 Phys. Plasmas 9 2149-55

[5] Connor J.W. et al 2004 Nucl. Fusion 44 R1-R49

[6] Ernst D.R. et al 2004 Phys. Plasmas 11 2637-48

[7] Wade M.R., Houlberg W.A., Baylor L.R., West W.P. and Baker D.R. 2001 J. Nucl. Mater. 290 773-7

[8] Takenaga H. et al 2003 Nucl. Fusion 43 1235-45

[9] Lao L.L., St John H., Stambaugh R.D., Kellman A.G. and Pfeiffer W. 1985 Nucl. Fusion 25 1611-22

[10] Beals D.F. et al 2005 Installation and operation of new long pulse DNB on Alcator C-Mod 21st IEEE/NPSS Symp. on
Fusion Engineering (Knoxville, TN, USA) (Piscataway, NJ: IEEE Nuclear and Plasma Sciences Society)

[11] Korepanov S.A. et al 2004 Rev. Sci. Instrum. $751829-31$

[12] Bespamyatnov I.O., Rowan W.L. and Granetz R.S. 2008 Compact, accurate description of diagnostic neutral beam (DNB) propagation and attenuation in a high temperature plasma for charge exchange recombination spectroscopy (CXRS) analysis Rev. Sci. Instrum. at press

[13] Rowan W.L., Bespamyatnov I.O. and Granetz R.S. 2008 Wide-view CXRS diagnostic for Alcator C-Mod Rev. Sci. Instrum. at press

[14] Bespamyatnov I.O., Rowan W.L., Granetz R.S. and Beals D.F. 2006 Rev. Sci. Instrum. 77 10F123

[15] Summers H.P. 2004 Atomic Data and Analysis Structure User Manual (Glasgow: University of Strathclyde)

[16] Anderson H. et al 2000 Plasma Phys. Control. Fusion 42 781-806

[17] Suzuki S. et al 1998 Plasma Phys. Control. Fusion 40 2097-111

[18] Zhurovich K. et al 2007 Nucl. Fusion 47 1220-31

[19] Dubuit N., Garbet X., Parisot T., Guirlet R. and Bourdelle C. 2007 Phys. Plasmas 14042301

[20] Horton W. and Rowan W. 1994 Phys. Plasmas 1 901-8

[21] Testa D. et al Nucl. Fusion 46 562-79

[22] Hulse R.A. 1983 Nucl. Technol./Fusion 3 259-72

[23] Wenzel K.W. and Sigmar D.J. 1990 Nucl. Fusion 30 1117-27

[24] Hirshman S.P. and Sigmar D.J. 1981 Nucl. Fusion 21 1079-201

[25] Chen H., Hawkes N.C., Ingesson L.C., Peacock N.J. and Haines M.G. 2001 Nucl. Fusion 41 31-46

[26] Takenaga H. 2001 Phys. Plasmas 8 2217-23

[27] Rice J.E. et al 2007 Fusion Sci. Technol. 51 357-68 